\title{
REVIEW
}

\section{Sweet talk: insights into the nature and importance of glucose transport in lung epithelium}

\author{
James P. Garnett, Emma H. Baker and Deborah L. Baines
}

ABSTRACT: For over 50 years, glucose has been recognised to cross the lung epithelial barrier and be transported by lung epithelial cells. However, until recently, research into these processes focused on their effects on lung liquid volume. Here, we consider a newly identified role for pulmonary glucose transport in maintaining low airway surface liquid (ASL) glucose concentrations and propose that this contributes to lung defence against infection.

Glucose diffuses into ASL via paracellular pathways at a rate determined by paracellular permeability and the transepithelial glucose gradient. Glucose is removed from ASL in proximal airways via facilitative glucose transporters, down a concentration gradient generated by intracellular glucose metabolism. In the distal lung, glucose transport via sodium-coupled glucose transporters predominates. These processes vary between species but universally maintain ASL glucose at 3-20-fold lower concentrations than plasma.

ASL glucose concentrations are increased in respiratory disease and by hyperglycaemia. Elevated ASL glucose in intensive care patients was associated with increased Staphylococcus aureus infection. Diabetic patients with and without chronic lung disease are at increased risk of respiratory infection. Understanding of mechanisms underlying lung glucose homeostasis could identify new therapeutic targets for control of ASL glucose and prevention and treatment of lung infection.

KEYWORDS: Airway epithelium, alveoli, facilitative glucose transporter, glucose, lung, sodiumcoupled glucose transporter

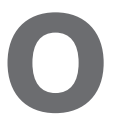
ur understanding of the role of glucose transport in the lung and the mechanisms that regulate glucose movement across the human lung epithelium lags far behind that of the gut and kidney. Since the mid-1960s, it has been known that there are energy-dependent, sodium-coupled glucose transporter (SGLT) and energy-independent, facilitative glucose transporter (GLUT) pathways for glucose uptake in the lung [1], and that glucose can permeate the alveolar epithelial barrier [2]. However, much subsequent research in this area was focused on the role of pulmonary SGLT transport as a modifier of lung liquid volume [3, 4], and the effects of starvation and diabetes on glucose transport $[1,5]$. It is only recently that we and others have begun to investigate glucose transport as an important mechanism for maintaining a nutrient-depleted environment in the lung lumen to limit the growth of pathogenic organisms.

\section{NORMAL GLUCOSE CONCENTRATIONS IN AIRWAY SURFACE LIQUID}

Glucose concentrations are 3-20 times lower in the fluid that lines the lung epithelium (airway surface liquid; ASL) than in plasma. This is in contrast to conditions in the gut and kidney, where luminal glucose concentrations regularly exceed plasma glucose concentrations [6, 7]. In humans, glucose concentrations were found to be $<1 \mathrm{mM}$ in nasal ASL [8] and mean \pm SD $0.4 \pm 0.2 \mathrm{mM}$ in the lower respiratory tract, 12.5 times lower than plasma concentrations, which are normally maintained at $\sim 5 \mathrm{mM}$ [9]. In a perfused, fluid-filled, adult rat lung model, ASL glucose was estimated to be $0.5 \mathrm{mM}$ when perfusate glucose was $10 \mathrm{mM}$ [4]. In a chronically catheterised sheep fetus between 122 and 143 days gestation, lung liquid glucose was $<0.01 \mathrm{mM}$ and plasma glucose $0.19 \mathrm{mM}$ [3]. In vitro studies in resistive human lung epithelial cell monolayers grown at an air-liquid interface have
AFFILIATIONS

Division of Biomedical Sciences St George's, University of London, London, UK.

CORRESPONDENCE

D.L. Baines

Division of Biomedical Sciences

St George's

University of London

Tooting

London

SW17 ORE

UK

E-mail: dbaines@sgul.ac.uk

Received:

March 282012

Accepted after revision:

July 192012

First published online:

Aug 092012 
supported these in vivo observations. In immortalised human airway cells (line H441) with $10 \mathrm{mM}$ glucose in the basolateral medium, apical ASL glucose was $0.24 \pm 0.07 \mathrm{mM}$ [10]. In primary cultures of human bronchial epithelial cells (HBECs) with $16.6 \pm 0.4 \mathrm{mM}$ glucose in the basolateral medium, ASL glucose was $2.2 \pm 0.5 \mathrm{mM}[11]$.

\section{PROCESSES THAT DETERMINE ASL GLUCOSE CONCENTRATIONS}

ASL glucose concentrations are the net result of diffusion of glucose from blood and interstitial fluid across the respiratory epithelium into the ASL, and removal of glucose from ASL by epithelial glucose transport processes. Glucose diffusion is determined by epithelial permeability to glucose, the transepithelial glucose gradient and surface area. Glucose removal is determined by cellular glucose uptake and metabolism (fig. 1) [4, 12-14].

\section{GLUCOSE DIFFUSION INTO ASL}

Movement of glucose from the blood and interstitial fluid into the ASL occurs primarily by paracellular pathways. Early in vivo studies found that rabbit respiratory epithelium and pig tracheal epithelium were permeable to L-glucose, an isoform of glucose that is neither transported nor metabolised. In rat lungs, increasing paracellular permeability with protamine increased luminal glucose concentrations $[15,16]$. More recent work has shown that L-glucose, applied to the basolateral side of resistive human airway epithelial cell monolayers grown on permeable filters at an air-liquid interface, moved into the apical compartment in a time- and concentration-dependent manner, consistent with paracellular diffusion [10-12]. Moreover, apical appearance of L-glucose increased exponentially with a decrease in transepithelial electrical resistance [10, 12]. These findings imply that glucose diffuses passively across the epithelium via paracellular pathways, and that this process is affected by the permeability of epithelial tight junctions to glucose and by the glucose concentration gradient (fig. 1).

\section{Paracellular pathways}

Tight junctions between epithelial cells predominantly determine the permeability of the epithelium [17]. They comprise transmembrane proteins, including junctional adhesion molecules, claudins and occludins, which are linked to cytoskeletal proteins and each other by scaffolding proteins, such as zonula occludens $(\mathrm{ZO})$ proteins. The protein components and their localisation contribute to the formation of different populations of size- and charge-limiting transcellular diffusion pathways. These pathways have been broadly classified into pore and leak types [18-22]. The pathway(s) by which glucose crosses the epithelial tight junction barrier are currently unknown.

ZO-1, occludin and claudins are the main junction proteins determining paracellular permeability [21, 22]. Claudins are critical for tight junction formation, affecting both pathways and charge selectivity of tight junction pores. In airway epithelial cells, differential expression of claudin-1, 3 and 5 alters epithelial permeability to large molecules. Claudin-2 is important for cation conductivity but has no effect on the flux of mannitol (which is similar in size to glucose) [23-25]. Overexpression of occludin increases epithelial permeability to small hydrophilic molecules while reducing ion movement [26]. The role of tight junctions in regulating glucose movement through epithelial tight junctions in the lung will undoubtedly be complex. However, identification of proteins and signalling pathways that restrict glucose movement could provide potential therapeutic targets to reduce glucose in ASL.

\section{Transcellular pathways}

There is some evidence that glucose can move by transcellular pathways from the blood and interstitial fluid into the ASL. Pezzulo et al. [11] compared bidirectional flux of L-glucose, which diffuses by paracellular pathways as it is not transported, and 2-deoxyglucose (2-DOG), which can be transported and moved by paracellular and transcellular pathways. 2-DOG flux in both the basolateral-to-apical and apical-tobasolateral directions was identified, indicating the presence of a)

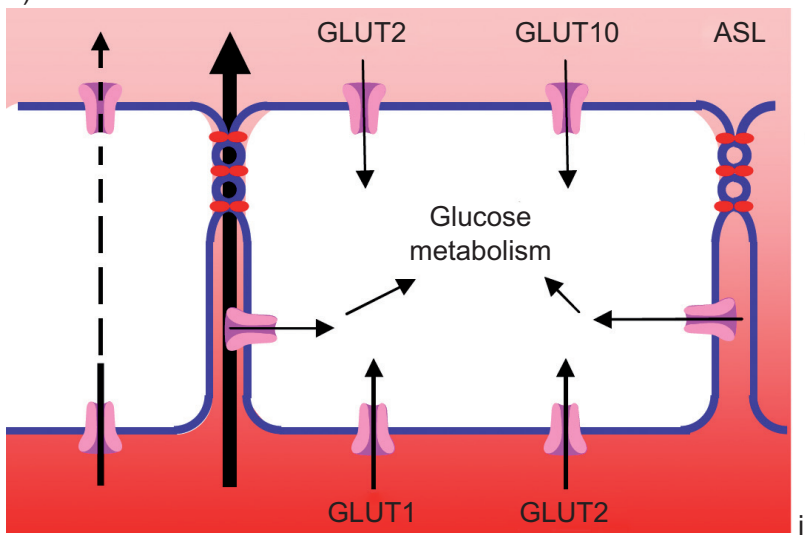

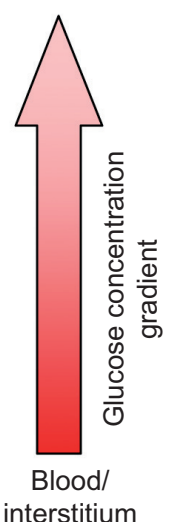

b)

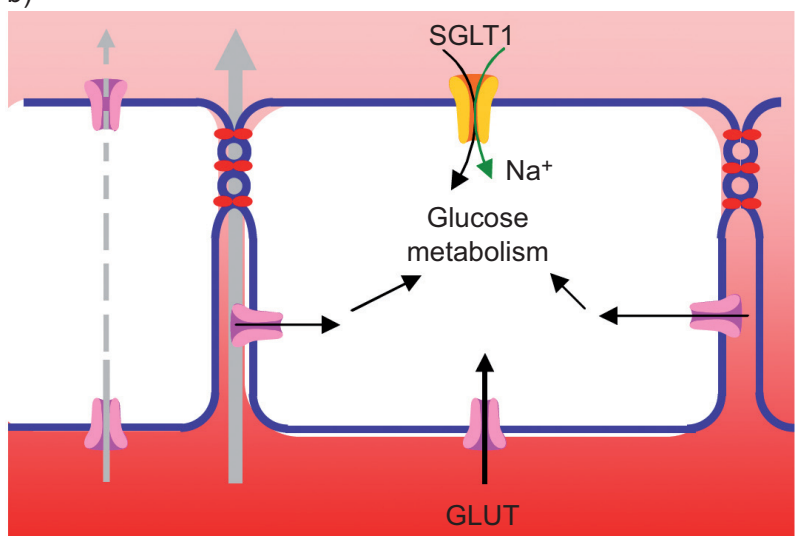

FIGURE 1. Current model of the mechanisms controlling glucose concentrations in the surface liquid lining the airway and distal lung epithelium. a) In the airway epithelium, the glucose concentration of the airway surface liquid (ASL) is the net effect of paracellular diffusion (and, to a lesser extent, the transcellular flux of glucose) from the blood and interstitial fluid across respiratory epithelium into the ASL, and removal of glucose from ASL by uptake into epithelial cells. Glucose uptake across the apical membrane is mediated by facultative glucose transporter (GLUT)2 and GLUT10, and across the basolateral membrane by GLUT2 and GLUT1. b) In the distal (alveolar) lung epithelium, glucose uptake across the apical membrane is via sodium-coupled glucose transporter (SGLT)1 and the basolateral membrane via unidentified GLUTs. 
transcellular pathways for glucose movement across airway epithelium (fig. 1).

\section{Transepithelial glucose gradient}

The airway epithelium maintains the glucose concentration gradient, with blood/interstitial glucose concentrations being higher than ASL glucose concentrations (see earlier) $[3,8,9]$. However, if this gradient is increased by elevation of blood and interstitial glucose concentrations, this increases movement of glucose into ASL. In healthy human volunteers, experimental elevation of blood glucose from $\sim 5 \mathrm{mM}$ to $12-15 \mathrm{mM}$ increased glucose in nasal ASL from $<1$ to $4.8 \pm 2.2 \mathrm{mM}$ [27] and in lower respiratory tract ASL from $0.36 \pm 0.27$ to $0.75 \pm 0.39 \mathrm{mM}$ [9]. In support of these findings, people with diabetes mellitus had elevated nasal (median $4 \mathrm{mM}$, interquartile range 2-7 $\mathrm{mM}$ ) [8] and lower respiratory tract $(1.2 \pm 0.7 \mathrm{mM})$ ASL glucose concentrations [9].

\section{THE ROLE OF GLUCOSE TRANSPORT IN LIMITING ASL GLUCOSE}

As glucose is able to diffuse across airway epithelium, with time, ASL glucose might be expected to equilibrate with blood glucose concentrations. As this is not the case, additional mechanisms must exist to remove glucose from the ASL and/or restrict diffusion and transcellular transport. In the next section, we will expand on the nature and role of glucose transport in lung epithelium.

\section{Glucose transporters}

Glucose transport across mammalian cell membranes is mediated either by facilitative GLUTs (encoded by the SLC2 gene family) or by SGLTs (SLC5 gene family). Glucose moves through GLUTs by passive diffusion down a concentration gradient generated by hexokinases (HKs) and glucokinases, which phosphorylate intracellular glucose. By contrast, SGLT-mediated transport is driven by sodium and glucose gradients and, therefore, can move glucose against its concentration gradient.

The human SLC2 family consists of 14 known isoforms (SLC2A1-14) encoding glucose transporter proteins, each with different tissue expression patterns, substrate (sugar) specificity and transport kinetics. According to their sequence homology, GLUTs can be divided into three distinct classes: class I comprises the well-characterised transporters GLUT1-4 [28-31] and GLUT14 (a gene duplication of GLUT3) [32]; class II includes the fructose transporter GLUT5 [33], and GLUT7 [34], GLUT9 [35] and GLUT11 [36]; class III comprises the recently identified transporters GLUT6 [33], GLUT8 [37], GLUT10 [38], GLUT12 [39] and the proton-driven human myoinositol transporter (GLUT13) [40]. Their affinities for glucose vary greatly. GLUT2 has a lower affinity for glucose than other members of the glucose transporter family (Michaelis constant $(\mathrm{Km})$ of $\sim 32 \mathrm{mM}$ when expressed in Xenopus oocytes) [41], while GLUT10 has the highest affinity $(\mathrm{Km}$ $\sim 0.3 \mathrm{mM}$ ) [42]. The human SLC5 gene family consists of 11 isoforms (SLC5A1-11), of which SLC5A1 (SGLT1) [43] and SLC5A2 (SGLT2) [44] are known to encode SGLTs.

\begin{tabular}{|c|c|c|c|c|c|c|c|c|c|c|}
\hline TABLE 1 & $\begin{array}{l}\text { Facultative } \\
\text { epithelial ce }\end{array}$ & $\begin{array}{l}\text { ose tran } \\
\text { human }\end{array}$ & $\begin{array}{l}\operatorname{ter}(\mathrm{GL} \\
\text { chial tis }\end{array}$ & $\begin{array}{l}\text { and sodi } \\
\text { and who }\end{array}$ & ing & cose tra & orter (S & ) express & in hum & cultured \\
\hline \multirow[t]{3}{*}{ Transporter } & \multicolumn{6}{|c|}{ Human cultured epithelial cells } & \multicolumn{2}{|c|}{ Bronchial tissue } & \multicolumn{2}{|c|}{ Whole lung } \\
\hline & \multicolumn{4}{|c|}{ H441 } & \multicolumn{2}{|c|}{ HBECs } & \multirow{2}{*}{ mRNA } & \multirow{2}{*}{ Protein } & \multirow{2}{*}{ mRNA } & \multirow{2}{*}{ Protein } \\
\hline & \multicolumn{2}{|c|}{ Nonpolarised } & \multicolumn{2}{|c|}{ Polarised } & mRNA & Protein & & & & \\
\hline \multicolumn{11}{|l|}{ GLUTs } \\
\hline GLUT1 & $+[13]$ & - [13] & & - [13] & $+[11]$ & $+[11]$ & $+[11]$ & $-[45]$ & $+[47]$ & $-[45,46]$ \\
\hline GLUT2 & $+[13]$ & $+[13]$ & & $+[10,13]$ & $+[11]$ & - [11] & $+[11]$ & $+[13,46]$ & & \\
\hline GLUT3 & $-[13]$ & $-[13]$ & & $-[13]$ & $+[11]$ & $-[11]$ & $+[11]$ & $-[45]$ & $+[47]$ & $-[45,46]$ \\
\hline GLUT8 & & & & & $+[11]$ & $-[11]$ & $+[11]$ & & & \\
\hline GLUT9 & & & & & $+[11]$ & & $+[11]$ & & & \\
\hline GLUT10 & & & & $+[10]$ & $+[11]$ & $+[11]$ & $+[11]$ & & $+[48]$ & \\
\hline GLUT11 & & & & & $+[11]$ & & $+[11]$ & & & \\
\hline GLUT12 & & & & & $+[11]$ & & $+[11]$ & & & \\
\hline \multicolumn{11}{|l|}{ SGLTs } \\
\hline SGLT1 & & & & & $+[11]$ & $-[11]$ & $+[11]$ & & $+[49]$ & \\
\hline SGLT2 & & & & & $+[11]$ & $-[11]$ & $+[11]$ & & $+[49]$ & \\
\hline
\end{tabular}


GLUT- and SGLT-mediated glucose transport have been well characterised in many tissues and cell types, although relatively little is known about their roles in the lung. Understanding of this is greatly complicated by regional, species and developmental differences in pulmonary glucose transport.

\section{Glucose transport in airway epithelium}

In human airway epithelium (trachea, bronchi and bronchioles), GLUTs are the predominant glucose transporter type expressed (tables 1 and 2). GLUT10 is present in immortalised human airway epithelial cells (H441) [10], and GLUT2 protein was detected in both apical and basolateral membranes of H441 and in epithelial cells in human bronchial biopsies [13]. In primary cultured HBECs, GLUT10 was present in the apical membrane but GLUT1 protein was detected in basolateral membrane [11]. In H441 cell monolayers, phloretin (an inhibitor of GLUTmediated transport) inhibited apical glucose uptake and increased appearance of D-glucose in the apical compartment [12]. In HBEC monolayers, 2-DOG (a substrate for GLUT but not SGLT transport) was taken up across the apical membrane [11]. Taken together, these findings indicate that apical glucose uptake through GLUTs plays an important role in maintaining low ASL glucose concentrations.

As GLUTs only transport glucose down its concentration gradient, this poses the question as to how GLUTs can maintain low ASL glucose concentrations. Two mechanisms have been proposed from recent research. First, glucose taken up into airway epithelial cells is metabolised rapidly [12]. This would maintain low intracellular glucose concentrations, providing a driving force for glucose uptake across basolateral and apical membranes. Rapid metabolism of glucose would also be predicted to limit transcellular movement of glucose that could occur given the basolateral-to-apical glucose concentration gradient. Secondly, glucose uptake from the basolateral membrane and intercellular clefts could modify glucose concentration in localised microdomains and decrease glucose concentration gradients close to routes of paracellular diffusion. In support of this, basolateral phloretin increases appearance of glucose in the apical compartment of polarised H441 cell monolayers [12]. In this scenario, combined paracellular and transcellular glucose movement would be less than cell glucose uptake, allowing generation of low ASL glucose concentrations [11, 12]. We also speculate that the different apical and basolateral localisation of GLUTs may play an important role in regulating glucose uptake from these apical and basolateral domains. For example, the low Km of GLUT10 compared with the higher Kms of GLUT1 and GLUT2 would preferentially favour glucose uptake from the apical side at low glucose concentrations. These findings indicate that airway epithelial GLUTs could be targeted to enhance depletion of glucose in ASL in respiratory disease. Moreover, it could be envisaged that genetic mutations that decrease glucose transporter function could lead to compromised airway glucose homeostasis and result in recurrent pulmonary or upper airway infections.

Both of these hypotheses predict that glucose metabolism in airway epithelial cells is a key driver of GLUT transport and determinant of ASL glucose concentrations. Once taken up into the cell, conversion of glucose to glucose-6-phosphate is

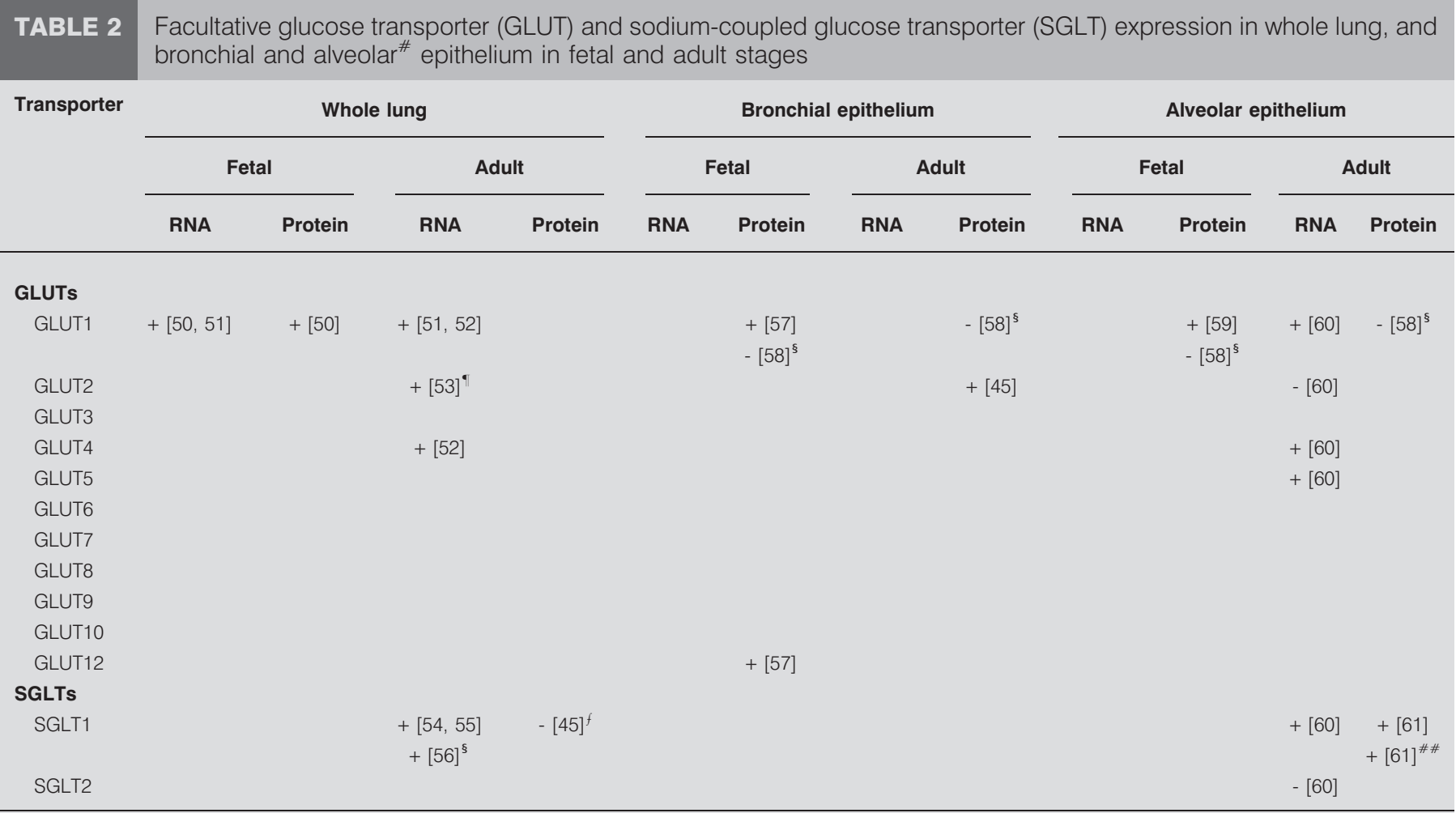


catalysed by HKs. As glucose phosphorylation is considered to be the rate-limiting step in glucose metabolism $[62,63]$ this is likely to be an important process in lung glucose homeostasis. HKI, HKII and HKIII have been identified in rat lung [52, 64], and HKII is present in human lung tumours and A549 alveolar epithelial cells $[65,66]$. How HK activity might regulate glucose uptake, transepithelial glucose transport and glucose concentrations in ASL has yet to be explored. However, it could be speculated that upregulation of HK activity would increase glucose uptake and reduce ASL glucose. There is already interest in activators and inhibitors of hexokinase activity for the treatment of diabetes. These enzymes may also provide potential therapeutic targets for the treatment and prevention of lung disease associated with elevated ASL glucose.

In human airway epithelium, sodium-coupled glucose transport does not appear to contribute to maintenance of low ASL glucose concentrations. SGLT protein has not been detected in human airway epithelium (table 1). In human tracheal epithelium mounted in Ussing chambers, short-circuit current (Isc) was not increased by luminal glucose or reduced by phlorizin (an SGLT inhibitor) [11]. There was no consistent effect of apically applied phlorizin on glucose uptake or Isc in human cell monolayers. Ussing chamber studies comparing sheep and human tracheal epithelium (from diseased lung) showed phlorizin produced a small reduction in Isc in ovine airways ( $~ 5 \%)$, but had no significant effect in human tissue [67].

While GLUTs dominate apical glucose transport in the surface epithelium of the airways, there is some enticing evidence that SGLTs could be present in the submucosal glands (SMGs). In polarised cultures of serous SMG cells (Calu-3 cells), the addition of phlorizin $(200 \mu \mathrm{M})$ inhibited $\sim 20 \%$ of basal Isc, which could be mimicked by removal of glucose from the apical bath solution [54], consistent with the presence of apical sodium-coupled glucose transport in these cells [68, 69]. Whether SGLTs are present in submucosal glands in vivo remains to be tested but it seems unlikely so far that they have a significant contribution to airway glucose transport given the lack of functional evidence (see earlier).

\section{Glucose transport in distal lung epithelium}

In distal lung (alveolar) epithelium, sodium-coupled glucose transport appears to be the major mechanism responsible for apical glucose uptake from the lumen. As studies have not been performed in human distal lung epithelium, understanding of this area comes from animal studies.

SGLT1, but not SGLT2, mRNA has been identified in distal lung epithelium from adult rats [55,60], and from mouse and human whole-lung tissue $[49,56]$. BODEGA et al. [61] recently identified SGLT protein in type I and II alveolar cells of rat and sheep lung. However, SGLT1 protein could not be detected in adult rabbit lung tissue. In rat, rabbit and sheep distal lung, glucose removal from the lumen is prevented by phlorizin, which inhibits SGLTs $[3,4,70]$. SGLT-mediated transport has been demonstrated in freshly isolated alveolar type II (ATII) cells from guinea pig [70]. In airway epithelial cells, the intracellular sodium concentration is low $(\sim 23 \mathrm{mM})$ compared with the sodium concentration in the ASL $(\sim 120 \mathrm{mM})$ or blood plasma $(\sim 140 \mathrm{mM})$ [71, 72]. SGLT utilises this sodium gradient to drive glucose uptake from ASL (low glucose) to the interstitium and blood (high glucose) against its concentration gradient.

By contrast, there is little evidence of luminal GLUT-mediated transport by the distal lung epithelium. Phloretin in the lung instillate did not inhibit glucose or fluid absorption from rabbit $[15,73]$ or rat whole lung [4], and there was little luminal 2DOG (GLUT-specific) uptake in fetal sheep lung [3]. However, both phlorizin and phloretin inhibit glucose uptake in isolated ATII cells from guinea pig lung [70], indicating that GLUTs could be present on the basolateral membrane.

The observation that airway and alveolar epithelium utilise diverse glucose transport mechanisms has led to speculation as to the role of apical glucose transport in different lung regions. In the airway, which is the first line of defence against infection, generation of low ASL glucose concentrations may be crucial for maintenance of airway sterility. In the distal lung, where gas exchange occurs, sodium-coupled glucose transport may be an important driver of fluid reabsorption and regulation of ASL volume, with generation of low ASL glucose concentrations as a secondary beneficial effect. Developmental changes in lung epithelial glucose transport also indicate differences in the role of these transport process in the fetal and adult lung.

\section{Developmental differences in lung glucose transport}

Developmental changes in lung glucose transporter expression have best been characterised in rat lung (table 2). In fetal rats, GLUT1 mRNA expression increases to maximal levels at gestational day 20 and falls to very low levels by postnatal day 8 [51]. Immunohistochemical staining of the rat fetus from gestational day 13 to 21 shows weak GLUT12 expression in the epithelial cell membrane of bronchioles at gestational day 19, which increases in intensity by day 21. Consistent with mRNA expression studies, GLUT1 protein was also observed in the airways at gestational day 19, decreasing in intensity at day 21 [57]. GLUT1 protein has also been detected in the alveolar epithelium of the fetal rat, although to date, GLUT1 protein has not been identified in either the airways or distal lung of the adult rat [59]. SGLT1 mRNA expression has been seen in the distal lung of both fetal and adult rats $[55,56]$.

In fetal sheep, the maximum rate of phlorizin-sensitive glucose uptake from the lung lumen increased with gestational age [3], indicating increased pulmonary expression of SGLTs towards birth. In humans, amniotic fluid glucose concentrations fall towards the end of pregnancy [74], consistent with increased pulmonary glucose reabsorption.

It is interesting to speculate on the role of glucose transport in fetal lungs. In fetal sheep, both addition of glucose to lung liquid [3] and maternal hyperglycaemia decrease net lung liquid secretion, probably by driving glucose-coupled sodium absorption. However as glucose concentrations are very low in normal fetal lung liquid, this process is unlikely to be a major determinant of lung sodium transport unless there is gestational diabetes. An alternative role could be to scavenge glucose from amniotic fluid, which could have the dual functions of conserving nutrients and reducing the risk of infection.

\section{Species and tissue differences in lung glucose transport}

The investigation of lung glucose transport in humans in vivo is challenging, so animal and cell culture models have been used 
to explore this field. There are important differences in the expression and function of transporters between species, cell types and experimental conditions that should be appreciated when choosing a model.

\section{Species differences}

Where it has been investigated, adults of most species have been found predominantly to express GLUT transporters in airway and SGLT in distal lung epithelium. Glucose uptake by isolated primary guinea pig ATII epithelial cells could be reduced both by phlorizin and by phloretin, indicating that not only SGLTs but also GLUTs are present in guinea pig distal lung cells [70]. By contrast, in adult sheep, glucose uptake across the tracheal epithelium can be inhibited by phlorizin and requires external sodium, consistent with sodium-coupled glucose transport [75]. Similarly, in isolated equine tracheae, phlorizin addition to the apical (but not the basolateral) membrane produced a significant decrease in Isc [76]. However, these differences between guinea pig and sheep/horse more likely reflect the experimental preparation. In intact epithelium, SGLT is predominantly present on the luminal membrane, but in isolated cells, there is a contribution from basolaterally localised GLUTs.

\section{Cell types}

Most in vitro studies of human airway epithelial glucose transport have used immortalised $\mathrm{H} 441$ cells or primary cultured HBECs. H441 cells derive from a papillary adenocarcinoma of the bronchiolar epithelium. When cultured at an airliquid interface, these cells form an absorptive epithelial monolayer, exhibit vectorial ion transport processes, and have similar morphological and phenotypic characteristics to human bronchiolar epithelium [77]. Both H441 cells and HBECs express GLUTs and do not exhibit SGLT expression. However, there may be differences in the GLUT isoform expressed, with H441 cells predominantly expressing GLUT2 and GLUT10 [10, 13] and HBECs expressing GLUT1 and GLUT10 [11].

\section{Experimental conditions}

Experimental conditions in both animal and in vitro studies may influence channel expression and function. In animals, investigation of distal lung glucose transport requires use of a fluidfilled lung model, which may alter transport processes. In cell culture, nonpolarised H441 cells express GLUT4, which was not detected in H441 cells polarised at an air-liquid interface [13].

\section{EFFECTS OF ELEVATED ASL GLUCOSE CONCENTRATIONS}

So what are the implications of lung glucose homeostasis for human health and disease? Clinical observation has shown that ASL glucose concentrations are normally $\sim 12.5$ times lower in human ASL than in plasma. However, ASL glucose concentrations are elevated in respiratory disease and when blood glucose is elevated. Nasal glucose concentrations are undetectable $(<1 \mathrm{mM})$ in healthy volunteers, but detectable in $90 \%$ of people with diabetes mellitus (median $4 \mathrm{mM}$, interquartile range 2 $7 \mathrm{mM}$ ) [8]. Ventilated patients on intensive care are more likely to have glucose detectable in bronchial aspirates when they have elevated blood glucose [7]. ASL glucose concentration, estimated using an exhaled breath condensate technique (breath glucose), was found to be $0.4 \pm 0.2 \mathrm{mM}$ in healthy volunteers but was elevated to $2.0 \pm 1.1 \mathrm{mM}$ in patients with cystic fibrosis,
$1.2 \pm 0.7 \mathrm{mM}$ in patients with diabetes mellitus and by the greatest amount $(4.0 \pm 2.0 \mathrm{mM})$ in people with both cystic fibrosis and diabetes mellitus [9]. In patients undergoing bronchoscopy, glucose was found to be elevated in bronchoalveolar lavage fluid (BAL) from patients with chronic obstructive pulmonary disease (COPD) and chronic severe asthma compared with levels in BAL fluid from healthy controls [78, 79]. These scenarios involve hyperglycaemia as a result of stress, and type I and type II diabetes mellitus. Any specific effect(s) of these individual disorders on glucose transport and homeostasis is, as yet, unclear. However, this may be important to understand, particularly with type II diabetes becoming more prevalent in the Western population.

There is both direct and indirect evidence that elevated ASL glucose concentrations promote lung infection. The respiratory pathogens Staphylococcus aureus and Pseudomonas aeruginosa both utilise glucose as a growth substrate [80]. Patients intubated and ventilated in an intensive care unit who have detectable glucose in bronchial aspirates are more likely to have methicillin-resistant $S$. aureus isolated from aspirates than those without detectable glucose [81]. Diabetes mellitus is a predisposing factor for nasal colonisation with S. aureus [82]. Patients with COPD who also have diabetes mellitus are more likely to have Gram-negative organisms cultured from their sputum than those without diabetes [83]. During acute exacerbations of COPD, increasing admission blood glucose concentrations are associated with increasing an likelihood of isolating $S$. aureus and multiple pathogens from sputum [79]. The growth of $P$. aeruginosa was found to be increased in the lungs of hyperglycaemic mice in vivo [11]. In humans with cystic fibrosis, coexisting diabetes mellitus is associated with an increased risk of infection with multiple antibiotic-resistant P. aeruginosa [84]. Cystic fibrosis patients with diabetes mellitus had more pulmonary exacerbations [85], which were less likely to respond to intravenous antibiotics [86], than those without diabetes.

\section{QUESTIONS FOR THE FUTURE}

While there are several mechanisms that regulate glucose homeostasis across the pulmonary epithelium, glucose transport undoubtedly plays a key role in maintaining a low glucose concentration in the ASL and, thus, restricting the growth of respiratory pathogens. This newly identified role for glucose transport raises some important questions, particularly regarding our classic understanding of GLUT-mediated transport. For example, is GLUT abundance regulated in response to changes in ASL glucose concentration? Why are SGLTs only detectable in the distal lung (SGLT can transport glucose against its concentration gradient, which would more efficiently reduce glucose in ASL)? In addition, in the gut, SGLTs are proposed to have an important role as glucose sensors with consequent upregulation of GLUT-mediated transport [87]. What are the roles of the different transporters, especially GLUT1, GLUT2 and GLUT10, in pulmonary glucose homeostasis? Is pulmonary glucose transport compromised in respiratory disease and diabetes (particularly in type II diabetes), and does this contribute to increased ASL glucose and infection? Finally, could we target glucose transport in the lung to ameliorate some of the consequences of respiratory disease? 


\section{STATEMENT OF INTEREST}

None declared.

\section{REFERENCES}

1 Chaisson CF, Massaro D. 2-Deoxy-D-glucose uptake by lung slices from fed and fasted rats. J Appl Physiol 1978; 44: 380-383.

2 Taylor AE, Guyton AC, Bishop VS. Permeability of the alveolar membrane to solutes. Circ Res 1965; 16: 353-362.

3 Barker PM, Boyd CA, Ramsden CA, et al. Pulmonary glucose transport in the fetal sheep. J Physiol 1989; 409: 15-27.

4 Saumon G, Martet G, Loiseau P. Glucose transport and equilibrium across alveolar-airway barrier of rat. Am J Physiol 1996; 270: L183-L190.

5 Fricke RF, Longmore WJ. Effects of insulin and diabetes on 2deoxy-D-glucose uptake by the isolated perfused rat lung. J Biol Chem 1979; 254: 5092-5098.

6 Ferraris RP, Yasharpour S, Lloyd KC, et al. Luminal glucose concentrations in the gut under normal conditions. Am J Physiol 1990; 259: G822-G837.

7 DeFronzo RA, Davidson JA, Del Prato S. The role of the kidneys in glucose homeostasis: a new path towards normalizing glycaemia. Diabetes Obes Metab 2012; 14: 5-14.

8 Philips BJ, Meguer JX, Redman J, et al. Factors determining the appearance of glucose in upper and lower respiratory tract secretions. Intensive Care Med 2003; 29: 2204-2210.

9 Baker EH, Clark N, Brennan AL, et al. Hyperglycemia and cystic fibrosis alter respiratory fluid glucose concentrations estimated by breath condensate analysis. J Appl Physiol 2007; 102: 1969-1975.

10 Garnett JP, Nguyen TT, Moffatt JD, et al. Pro-inflammatory mediators disrupt glucose homeostasis in airway surface liquid. J Immunol 2012; 189: 373-380.

11 Pezzulo AA, Gutierrez J, Duschner KS, et al. Glucose depletion in the airway surface liquid is essential for sterility of the airways. PLoS One 2011; 6: e16166.

12 Kalsi KK, Baker EH, Fraser O, et al. Glucose homeostasis across human airway epithelial cell monolayers: role of diffusion, transport and metabolism. Pflugers Arch 2009; 457: 1061-1070.

13 Kalsi KK, Baker EH, Medina RA, et al. Apical and basolateral localisation of GLUT2 transporters in human lung epithelial cells. Pflugers Arch 2008; 456: 991-1003.

14 Pezzulo AA, Starner TD, Scheetz TE, et al. The air-liquid interface and use of primary cell cultures are important to recapitulate the transcriptional profile of in vivo airway epithelia. Am J Physiol Lung Cell Mol Physiol 2011; 300: L25-L31.

15 Wangensteen D, Bartlett M. D- and L-glucose transport across the pulmonary epithelium. J Appl Physiol 1984; 57: 1722-1730.

16 Saumon G, Martet G. Effect of changes in paracellular permeability on airspace liquid clearance: role of glucose transport. Am J Physiol 1996; 270: L191-L198.

17 Farquhar MG, Palade GE. Junctional complexes in various epithelia. J Cell Biol 1963; 17: 375-412.

18 Balda MS, Matter K. Tight junctions at a glance. J Cell Sci 2008; 121: 3677-3682.

19 Shimizu M, Fukunaga Y, Ikenouchi J, et al. Defining the roles of beta-catenin and plakoglobin in LEF/T-cell factor-dependent transcription using $\beta$-catenin/plakoglobin-null F9 cells. Mol Cell Biol 2008; 28: 825-835.

20 Ikenouchi J, Sasaki H, Tsukita S, et al. Loss of occludin affects tricellular localization of tricellulin. Mol Biol Cell 2008; 19: 4687-4693.

21 Shen L, Weber CR, Raleigh DR, et al. Tight junction pore and leak pathways: a dynamic duo. Annu Rev Physiol 2011; 73: 283-309.

22 Van Itallie CM, Holmes J, Bridges A, et al. The density of small tight junction pores varies among cell types and is increased by expression of claudin-2. J Cell Sci 2008; 121: 298-305.
23 Coyne CB, Gambling TM, Boucher RC, et al. Role of claudin interactions in airway tight junctional permeability. Am J Physiol Lung Cell Mol Physiol 2003; 285: L1166-L1178.

24 Coyne CB, Vanhook MK, Gambling TM, et al. Regulation of airway tight junctions by proinflammatory cytokines. Mol Biol Cell 2002; 13: 3218-3234.

25 Amasheh S, Meiri N, Gitter AH, et al. Claudin-2 expression induces cation-selective channels in tight junctions of epithelial cells. J Cell Sci 2002; 115: 4969-4976.

26 Aijaz S, Balda M, Matter K. Tight junctions: molecular architecture and function. Int Rev Cytol 2006; 248: 261-298.

27 Wood DM, Brennan AL, Philips BJ, et al. Effect of hyperglycaemia on glucose concentration of human nasal secretions. Clin Sci (Lond) 2004; 106: 527-533.

28 Mueckler M, Caruso C, Baldwin S, et al. Sequence and structure of a human glucose transporter. Science 1985; 229: 941-945.

29 Fukumoto $\mathrm{H}$, Seino S, Imura $\mathrm{H}$, et al. Sequence, tissue distribution, and chromosomal localization of mRNA encoding a human glucose transporter-like protein. Proc Natl Acad Sci USA 1988; 85: 5434-5438.

30 Kayano T, Fukumoto H, Eddy RL, et al. Evidence for a family of human glucose transporter-like proteins. Sequence and gene localization of a protein expressed in fetal skeletal muscle and other tissues. J Biol Chem 1988; 263: 15245-15248.

31 James DE, Strube M, Muecdler M. Molecular cloning and characterization of an insulin-regulatable glucose transporter. Nature 1989; 338: 83-87.

$32 \mathrm{Wu}$ X, Freeze HH. GLUT14, a duplicon of GLUT3, is specifically expressed in testis as alternative splice forms. Genomics 2002; 80: 553-557.

33 Kayano $\mathrm{T}$, Burant CF, Fukumoto $\mathrm{H}$, et al. Human facilitative glucose transporters. Isolation, functional characterization, and gene localization of cDNAs encoding an isoform (GLUT5) expressed in small intestine, kidney, muscle, and adipose tissue and an unusual glucose transporter pseudogene-like sequence (GLUT6). J Biol Chem 1990; 265: 13276-13282.

34 Li Q, Manolescu A, Ritzel M, et al. Cloning and functional characterization of the human GLUT7 isoform SLC2A7 from the small intestine. Am J Physiol Gastrointest Liver Physiol 2004; 287: G236-G242.

35 Phay JE, Hussain HB, Moley JF. Cloning and expression analysis of a novel member of the facilitative glucose transporter family, SLC2A9 (GLUT9). Genomics 2000; 66: 217-220.

36 Doege H, Bocianski A, Scheepers A, et al. Characterization of human glucose transporter (GLUT) 11 (encoded by SLC2A11), a novel sugar-transport facilitator specifically expressed in heart and skeletal muscle. Biochem J 2001; 359: 443-449.

37 Doege H, Schürmann A, Bahrenberg G, et al. GLUT8, a novel member of the sugar transport facilitator family with glucose transport activity. J Biol Chem 2000; 275: 16275-16280.

38 McVie-Wylie AJ, Lamson DR, Chen YT. Molecular cloning of a novel member of the GLUT family of transporters, SLC2A10 (GLUT10), localized on chromosome 20q13.1: a candidate gene for NIDDM susceptibility. Genomics 2001; 72: 113-117.

39 Rogers S, Macheda ML, Docherty SE, et al. Identification of a novel glucose transporter-like protein-GLUT-12. Am J Physiol Endocrinol Metab 2002; 282: E733-E738.

40 Augustin R. The protein family of glucose transport facilitators: it's not only about glucose after all. IUBMB Life 2010; 62: 315-333.

41 Buchs $\mathrm{A}, \mathrm{Wu} \mathrm{L}$, Morita $\mathrm{H}$, et al. Two regions of GLUT 2 glucose transporter protein are responsible for its distinctive affinity for glucose. Endocrinology 1995; 136: 4224-4230.

42 Zhao FQ, Keating AF. Functional properties and genomics of glucose transporters. Curr Genomics 2007; 8: 113-128.

43 Hediger MA, Coady MJ, Ikeda TS, et al. Expression cloning and cDNA sequencing of the $\mathrm{Na}^{+}$/glucose co-transporter. Nature 1987; 330: 379-381.

44 Wells RG, Mohandas TK, Hediger MA. Localization of the $\mathrm{Na}^{+}$/ glucose cotransporter gene SGLT2 to human chromosome 16 close to the centromere. Genomics 1993; 17: 787-789. 
45 Devaskar SU, deMello DE. Cell-specific localization of glucose transporter proteins in mammalian lung. J Clin Endocrinol Metab 1996; 81: 4373-4378.

46 Ito T, Noguchi Y, Satoh S, et al. Expression of facilitative glucose transporter isoforms in lung carcinomas: its relation to histologic type, differentiation grade, and tumor stage. Mod Pathol 1998; 11: 437-443.

47 Kurata $\mathrm{T}$, Oguri $\mathrm{T}$, Isobe $\mathrm{T}$, et al. Differential expression of facilitative glucose transporter (GLUT) genes in primary lung cancers and their liver metastases. Jpn J Cancer Res 1999; 90: 1238-1243.

48 Dawson PA, Mychaleckyj JC, Fossey SC, et al. Sequence and functional analysis of GLUT10: a glucose transporter in the type 2 diabetes-linked region of chromosome 20q12-13.1. Mol Genet Metab 2001; 74: 186-199.

49 Ishikawa N, Oguri T, Isobe T, et al. SGLT gene expression in primary lung cancers and their metastatic lesions. Jpn J Cancer Res 2001; 92: 874-879.

50 Simmons RA, Flozak AS, Ogata ES. Glucose regulates GLUT 1 function and expression in fetal rat lung and muscle in vitro. Endocrinology 1993; 132: 2312-2318.

51 Werner H, Adamo M, Lowe WL Jr, et al. Developmental regulation of rat brain/Hep G2 glucose transporter gene expression. Mol Endocrinol 1989; 3: 273-279.

52 Allen CB, Guo XL, White CW. Changes in pulmonary expression of hexokinase and glucose transporter mRNAs in rats adapted to hyperoxia. Am J Physiol 1998; 274: L320-L329.

53 Zuo J, Huang Z, Zhi A, et al. Cloning and distribution of facilitative glucose transporter 2 (SLC2A2) in pigs. Asian-Aust J Anim Sci 2010; 23: 1159-1165.

54 Singh M, Krouse M, Moon S, et al. Most basal IsC in Calu-3 human airway cells is bicarbonate-dependent $\mathrm{Cl}^{-}$secretion. Am J Physiol Lung Cell Mol Physiol 1997; 272: L690-L698.

55 Lee WS, Kanai Y, Wells RG, et al. The high affinity $\mathrm{Na}^{+} /$glucose cotransporter. Re-evaluation of function and distribution of expression. J Biol Chem 1994; 269: 12032-12039.

56 Icard P, Saumon G. Alveolar sodium and liquid transport in mice. Am J Physiol 1999; 277: L1232-L1238.

57 Macheda ML, Kelly DJ, Best JD, et al. Expression during rat fetal development of GLUT12 - a member of the class III hexose transporter family. Anat Embryol (Berl) 2002; 205: 441-452.

58 Mantych G, Devaskar U, deMello D, et al. GLUT 1 - glucose transporter protein in adult and fetal mouse lung. Biochem Biophys Res Commun 1991; 180: 367-373.

59 Hart CD, Flozak AS, Simmons RA. Modulation of glucose transport in fetal rat lung: a sexual dimorphism. Am J Respir Cell Mol Biol 1998; 19: 63-70.

60 Mamchaoui K, Makhloufi Y, Saumon G. Glucose transporter gene expression in freshly isolated and cultured rat pneumocytes. Acta Physiol Scand 2002; 175: 19-24.

61 Bodega F, Sironi C, Armilli M, et al. Evidence for $\mathrm{Na}^{+}$-glucose cotransporter in type I alveolar epithelium. Histochem Cell Biol 2010; 134: 129-136.

62 Salotra PT, Singh VN. Regulation of glucose metabolism in the lung: hexokinase-catalyzed phosphorylation, a rate-limiting step. Life Sci 1982; 31: 791-794.

63 Salotra PT, Singh VN. Regulation of glucose metabolism in rat lung: subcellular distribution, isozyme pattern, and kinetic properties of hexokinase. Arch Biochem Biophys 1982; 216: 758-764.

64 Katzen HM, Soderman DD, Cirillo VJ. Tissue distribution and physiological significance of multiple forms of hexokinase. Ann NY Acad Sci 1968; 151: 351-358.

65 Riddle SR, Ahmad A, Ahmad S, et al. Hypoxia induces hexokinase II gene expression in human lung cell line A549. Am J Physiol Lung Cell Mol Physiol 2000; 278: L407-L416.

66 Mamede M, Higashi $\mathrm{T}$, Kitaichi $\mathrm{M}$, et al. $\left[{ }^{18} \mathrm{~F}\right] \mathrm{FDG}$ uptake and PCNA, GLUT-1, and hexokinase-II expressions in cancers and inflammatory lesions of the lung. Neoplasia 2005; 7: 369-379.
67 Steel DM, Graham A, Geddes DM, et al. Characterization and comparison of ion transport across sheep and human airway epithelium. Epithelial Cell Biol 1994; 3: 24-31.

68 Lee MC, Penland CM, Widdicombe JH, et al. Evidence that Calu-3 human airway cells secrete bicarbonate. Am J Physiol Lung Cell Mol Physiol 1998; 274: L450-L453.

69 Krouse ME, Talbott JF, Lee MM, et al. Acid and base secretion in the Calu-3 model of human serous cells. Am J Physiol Lung Cell Mol Physiol 2004; 287: L1274-L1283.

70 Kemp PJ, Boyd CA. Pathways for glucose transport in type II pneumocytes freshly isolated from adult guinea pig lung. Am J Physiol 1992; 263: L612-L616.

71 Willumsen NJ, Boucher RC. Sodium transport and intracellular sodium activity in cultured human nasal epithelium. Am J Physiol 1991; 261: C319-C331.

72 Knowles MR, Robinson JM, Wood RE, et al. Ion composition of airway surface liquid of patients with cystic fibrosis as compared with normal and disease-control subjects. J Clin Invest 1997; 100: 2588-2595.

73 Basset G, Crone C, Saumon G. Significance of active ion transport in transalveolar water absorption: a study in isolated rat lung. J Physiol 1987; 384: 311-324.

74 Weiss PA, Hofmann H, Winter R, et al. Amniotic fluid glucose values in normal and abnormal pregnancies. Obstet Gynecol 1985; 65: 333-339.

75 Charon JP, McCormick J, Mehta A, et al. Characterization of sodium-dependent glucose transport in sheep tracheal epithelium. Am J Physiol 1994; 267: L390-L397.

76 Joris L, Quinton P. Evidence for electrogenic Na-glucose cotransport in tracheal epithelium. Pflügers Arch 1989; 415: 118-120.

77 Hermanns MI, Unger RE, Kehe K, et al. Lung epithelial cell lines in coculture with human pulmonary microvascular endothelial cells: development of an alveolo-capillary barrier in vitro. Lab Invest 2004; 84: 736-752.

78 Baker E, Akunuri S, Morgan M, et al. Effect of airways inflammation and prednisolone treatment on BAL glucose concentrations in asthma and COPD patients. Thorax 2008; 63: Suppl. 7, A40.

79 Baker EH, Janaway CH, Philips BJ, et al. Hyperglycaemia is associated with poor outcomes in patients admitted to hospital with acute exacerbations of chronic obstructive pulmonary disease. Thorax 2006; 61: 284-289.

80 Brennan A, Baines D, Woollhead A, et al. Development of an in vitro model to investigate the effect of glucose on the interaction between respiratory epithelia and bacterial pathogens. Thorax 2006; 61: Suppl. 2, ii101.

81 Philips BJ, Redman J, Brennan A, et al. Glucose in bronchial aspirates increases the risk of respiratory MRSA in intubated patients. Thorax 2005; 60: 761-764.

82 Tamer A, Karabay O, Ekerbicer H. Staphylococcus aureus nasal carriage and associated factors in type 2 diabetic patients. Jpn J Infect Dis 2006; 59: 10-14.

83 Loukides S, Polyzogopoulos D. The effect of diabetes mellitus on the outcome of patients with chronic obstructive pulmonary disease exacerbated due to respiratory infections. Respiration 1996; 63: 170-173.

84 Merlo CA, Boyle MP, Diener-West M, et al. Incidence and risk factors for multiple antibiotic-resistant Pseudomonas aeruginosa in cystic fibrosis. Chest 2007; 132: 562-568.

85 Jarad NA, Giles K. Risk factors for increased need for intravenous antibiotics for pulmonary exacerbations in adult patients with cystic fibrosis. Chron Respir Dis 2008; 5: 29-33.

86 Parkins MD, Rendall JC, Elborn JS. Incidence and risk factors for pulmonary exacerbation treatment failures in cystic fibrosis patients chronically infected with Pseudomonas aeruginosa. Chest 2012; 141: 485-493.

87 Kellett GL, Brot-Laroche E. Apical GLUT2: a major pathway of intestinal sugar absorption. Diabetes 2005; 54: 3056-3062. 